\title{
In celebration: The 40th birthday of the Pill
}

"The future is not likely to be dull". This statement, made by the Family Planning Association (now known as fpa) in $1960^{1}$ when it launched the first UK trial of the oral contraceptive pill, proved to be more than accurate! Four decades on from the introduction of 'the Pill' in 1961 (1960 in the US), history graphically illustrates how oral contraception not only revolutionised the field of birth control, but also fundamentally changed and improved the role and health of women, legitimised the involvement of the medical profession in the field of family planning, and split the Catholic Church.

Creation of the oral contraceptive pill followed more than 30 years of research, from early work by Haberlandt in the 1920s who demonstrated that fertility could be influenced by hormonal manipulation, to the pioneering work of researchers such as Marker, Djerassi, Rock and Pincus from the 1930s to the 1960s. The introduction of the Pill was greeted with enormous hype and expectation contraceptive methods were few in the 1960 s, something we forget with so many choices in 2001. The Lancet $^{2}$ discussed it as 'a subject second in importance only to the nuclear bomb'. Euphoria, however, soon gave way to concerns about safety, which resulted in three of the world's largest and most influential oral contraception research studies being set up - The RCGP Oral Contraception Study and The Oxford/FPA Contraceptive Study in the UK and the Nurses Health Study in the US. These studies, and the many hundreds that have followed, are fundamental to our understanding of the true risks, benefits and uncertainties of oral contraception.

Forty years on and oral contraceptive pills are among the most thoroughly researched drugs of all time - rightfully so, for a product that is used by young, healthy women for potentially long periods of time. Their safety profile needs to be exemplary. No contraceptive method is $100 \%$ effective with no side effects. This ideal is likely to be elusive, but development does not stand still. Research is vital in contributing to our knowledge but, and it is an important but, how research is carried out, discussed and reported can have the most devastating effect on the way the Pill is perceived, provided, used and developed.

The Pill is not perfect, it will not suit everyone, but today's oral contraceptive pills bear no resemblance to, and should not be compared with, their early predecessors. Research has provided a much clearer picture about risks and benefits. This in turn has meant that informed medical practice is more careful in its selection of users. We can be confident that, when trained and up to date professionals appropriately prescribe the Pill to women who are nonsmokers, with no medical contraindications, there are negligible risks - fewer than seen in pregnancy and childbirth. Yet 40 years on, and although widely used, the Pill is not universally popular. It continues to be one of the most worried over and talked about drugs, regularly making newspaper headlines. Consequently, women's fears persist out of all proportion to actual risk, and in most cases they are unaware of the Pill's substantial non-contraceptive health benefits. Confidence in the method is low and this is related to poor Pill taking, inconsistent use, missed Pills, incorrect transition between Pill packs and early discontinuation. Professionals' knowledge of the Pill's advantages and disadvantages remains varied. The mistrust and ignorance surrounding the Pill, by both women and health professionals, is surely unacceptable.

In celebrating the $40^{\text {th }}$ birthday of the Pill, we can say it has without doubt lived up to those early expectations of being a highly effective and safe method of fertility control for women. If it is to continue to be a valid contraceptive choice for the future, we have to stop being complacent and work much harder to improve and promote women's general understanding and confidence in oral contraception. We must recognise the need to unravel and counter the myths, misconceptions and misinformation that continually surround oral contraception. This means improving practice, communication and the availability of accurate (evidence-based), complete, consistent and memorable information.

The next 40 years are likely to see major advances in contraception; the Pill has already paved the way for new and innovative hormonal delivery systems for women and now, men. Confidence in today's methods is vital for acceptance of tomorrow's.

Toni Belfield, BSC (Hons), FRSH

Director of Information, fpa, London, $U K$

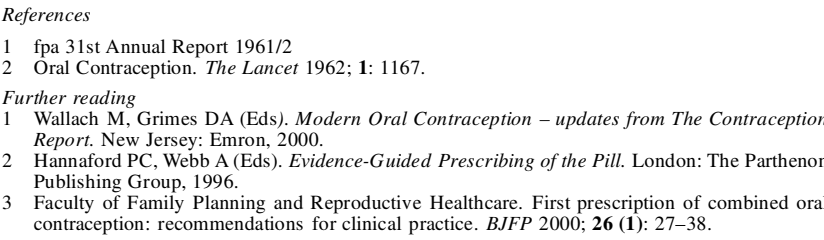

\section{Progress for the Journal of Family Planning and Reproductive Health Care}

\section{Structured abstracts}

From the July 2001 (Volume 27, Number 3) the Journal is changing from summaries to publishing structured abstracts for all papers. This is in line with other major peer review journals. 
conclusions. Review and meta-analysis abstracts should be structured under headings such as objective, data sources, study selection, data extraction, data synthesis and conclusion. Consensus statements should be structured under headings such as objective, participants, evidence, consensus process and conclusions. Observation manuscripts should be no longer than 200 words and described under headings such as background, observations and conclusions.

No information should be reported in the abstract that does not appear in the body of the text. The abstract should not contain references. It should emphasise new and important information that the study or investigation brings to the topic area. Advice on preparing structured abstracts can be found on the website http://jama.amaassn.org/info/auinst_abs.html.

\section{Committee on Publication Ethics (COPE) - Guidelines on good publication ethics}

COPE was founded in 1997 by a group of British medical editors, including those of the BMJ, Gut and Lancet, as a response to growing anxiety about the integrity of authors and the ethics of the publication process. The COPE guidelines on good publication ethics were published in 1999. The Journal Editorial Advisory Board has decided to adopt these guidelines. There are 10 topic areas, some of which will be published in the Journal over the next three issues. The full text can be found on http://www. publicationethics.org.uk

\section{Study design and ethical approval Definition}

Good research should be well justified, well planned, appropriately designed, and ethically approved. To conduct research to a lower standard may constitute misconduct.

\section{Action}

1 Laboratory and clinical research should be driven by protocol; pilot studies should have a written rationale.

2 Research protocols should seek to answer specific questions, rather than just collect data.

3 Protocols must be carefully agreed by all contributors and collaborators, including, if appropriate, the participants.

4 The final protocol should form part of the research record.

5 Early agreement on the precise roles of the contributors and collaborators, and on matters of authorship and publication, is advised.

6 Statistical issues should be considered early in the study design, including power calculations, to ensure that there are neither too few nor too many participants.

7 Formal and documented ethical approval from an appropriately constituted research ethics committee is required for all studies involving people, medical reports, and anonymised human tissues.

8 Use of human tissues in research should conform to the highest ethical standards, such as those recommended by the Nuffield Council on Bioethics.

9 Fully informed consent should always be sought. It may not always be possible, however, and in such circumstances, an appropriately constituted research ethics committee should decide if this is ethically acceptable.

10 Where participants are unable to give fully informed consent, research should follow international guidelines, such as those of the Council for International Organizations of Medical Sciences (CIOMS).

11 Animal experiments require full compliance with local, national, ethical, and regulatory principles, and local licensing arrangements. International standards vary.

12 Formal supervision, usually the responsibility of the principle investigator, should be provided for all research projects; this must include quality control, and the frequent review and long-term retention (may be up to 15 years) of all records and primary outputs.

\section{Data analysis \\ Definition}

Data should be appropriately analysed, but inappropriate analysis does not necessarily amount to misconduct. Fabrication and falsification of data do constitute misconduct.

\section{Action}

1 All sources and methods used to obtain and analyse data, including any electronic pre-processing, should be fully disclosed; detailed explanations should be provided for any exclusions.

2 Methods of analysis must be explained in detail, and referenced, if they are not in common use.

3 The post hoc analysis of subgroups is acceptable, as long as this is disclosed. Failure to disclose that the analysis was post hoc is unacceptable.

4 The discussion section of a paper should mention any issues of bias which have been considered, and explain how they have been dealt with in the design and interpretation of the study.

\section{Authorship \\ Definition}

There is no universally agreed definition of authorship, although attempts have been made. As a minimum, authors should take responsibility for a particular section of the study.

\section{Action}

1 The award of authorship should balance intellectual contributions to the conception, design, analysis and writing of the study against the collection of data and other routine work. If there is no task than can reasonably be attributed to a particular individual, then that individual should not be credited with authorship.

2 To avoid disputes over attribution of academic credit, it is helpful to decide early on in the planning of a research project who will be credited as authors, as contributors, and who will be acknowledged.

3 All authors must take public responsibility for the content of their paper. The multidisciplinary nature of much research can make this difficult, but this can be resolved by the disclosure of individual contributions.

4 Careful reading of the target journal's 'Advice to Authors' is advised, in the light of current uncertainties.

Fran Reader, FRCOG, MFFP

Honorary Editor Journal of Family Planning and Reproductive Health Care 